\title{
The Temperature of Morality: A Behavioral Study Concerning the Effect of Moral Decisions on Facial Thermal Variations in Video Games
}

\author{
Gianluca Guglielmo* \\ Michal Klincewicz* \\ g.guglielmo@tilburguniversity.edu \\ m.w.klincewicz@uvt.nl \\ Cognitive Science and Artificial Intelligence, Tilburg University \\ Tilburg, Netherlands
}

\begin{abstract}
In this paper, we report on an experiment with The Walking Dead (TWD), which is a narrative-driven adventure game with morally charged decisions set in a post-apocalyptic world filled with zombies. This study aimed to identify physiological markers of moral decisions and non-moral decisions using infrared thermal imaging (ITI). ITI is a non-invasive tool used to capture thermal variations due to blood flow in specific body regions that might be caused by sympathetic activity. Results show that moral decisions seem to elicit a significant decrease in temperature in the chin region 20 seconds after participants are presented with a moral decision. However, given the small sample involved, and the lack of significance in other regions, future studies might be needed to confirm the results obtained in this work.
\end{abstract}

\section{CCS CONCEPTS}

- Applied computing $\rightarrow$ Psychology; • Human-centered computing $\rightarrow$ Empirical studies in HCI; Laboratory experiments.

\section{KEYWORDS}

Video Games, Moral Psychology, Moral Foundations Theory, Infrared Thermal Imaging, Moral Decision-Making

\section{ACM Reference Format:}

Gianluca Guglielmo and Michal Klincewicz. 2021. The Temperature of Morality: A Behavioral Study Concerning the Effect of Moral Decisions on Facial Thermal Variations in Video Games. In The 16th International Conference on the Foundations of Digital Games (FDG) 2021 (FDG'21), August 3-6, 2021, Montreal, QC, Canada. ACM, New York, NY, USA, 4 pages. https://doi.org/10.1145/3472538.3472582

\section{INTRODUCTION}

Until recently, moral decision-making has been often investigated with experimental paradigms from behavioral economics and game theory, such as the prisoner dilemma, the ultimatum game [7],

*Both authors contributed equally to this research.

\section{(c) (†) (2)}

This work is licensed under a Creative Commons

Attribution-NonCommercial-ShareAlike International 4.0 License.

FDG'21, August 3-6, 2021, Montreal, QC, Canada

(c) 2021 Copyright held by the owner/author(s).

ACM ISBN 978-1-4503-8422-3/21/08.

https://doi.org/10.1145/3472538.3472582 and the trolley dilemma [14, 27]. Despite their extensive use, these paradigms are a relatively poor analogue of moral decision-making, since they involve betting and probability calculations, neither of which is a necessary part of the process of making a moral judgment and which can strip away the informational context that often plays a crucial role in determining individual moral judgments. These contextual features can be represented in the digital environment of a video game [24]. For this reason, moral scenarios presented in video games might be more like those encountered in real life [25].

Morally charged games have features that make them distinct from non-morally charged ones. In typical cases, these features are likely to invoke a variety of emotional reactions in the players themselves, as demonstrated by several studies focusing on sympathetic physiological responses $[5,28]$ and brain regions involved in the processing of morally charged stimuli $[2,21]$. Physiological changes that result from morally charged stimuli can also be detected by measures of thermal variations in the skin using infrared thermal imaging (ITI).

Our aim here is to take these insights and methods and apply them to morally charged decisions within The Walking Dead. More specifically, this study investigates the way in which temperature in participants' faces changes when they are faced with decisions that are morally charged. Results obtained shed light on the effect of morally charged stimuli on thermal variation and the future use of video games to investigate moral decision-making.

\section{RELATED WORK}

\subsection{Moral Foundations Theory}

For this specific study, moral foundation theory (MFT) was used because of its application in social and moral psychology [9, 13]. MFT assumes that moral decision-making is shaped at least in part by evolution and subsequently modified by cultural influence [15] but ultimately executed by mechanisms, which embody the five 5 moral foundations [12]: Harm/Care ; Fairness/Cheating; Loyalty/Betrayal; Authority/Subversion [1]; Sanctity/Degradation [22].

Whether these foundations are the only ones, or whether they are in some ways overlapping, continues to be a source of disagreement in moral psychology. For example, Graham et al. suggested there may exist a sixth Liberty/Oppression foundation [12]. MFT has also been criticized for the lack of explanation about the different degrees of cooperation between individuals and groups [8] and its moral relativism [11]. Nonetheless, despite criticisms and 
downsides, MFT is often used to operationalize and explain human moral decision-making today [6].

\subsection{Sympathetic activity and moral stimuli}

There is mounting evidence that moral decision-making involves the autonomic nervous system and specifically the sympathetic nervous system [5,28]. This is perhaps unsurprising since the sympathetic nervous system is in charge of coping with stressful events and is responsible for activating the flight-or-fight response [26]; moral decisions can sometimes be stressful. The fight-or-flight response that often accompanies stressful events is mediated by the release of noradrenaline and the subsequent release of adrenaline from the adrenal medulla [17]. Both of these neurotransmitters play a crucial role in changing heart rate, breathing, and can induce sweating, which is implicated in increased skin galvanic response. Noradrenaline mediates the activity in regions of the limbic system, which play a role in emotional processing and emotional learning [26]. These assumptions also found evidence in other independent neuroscientific and psychopharmacological studies [17].

\subsection{Infrared Thermal Imaging}

ITI can be used to monitor thermal variations due to sympathetic activity [10] on the assumption that this activity indirectly influences thermal variations in the skin [28]. For example, moral violations, such as lying, correlate with an increase in temperature in the nasal region [19]; while stressful stimuli affect the chin region [10]. This in turn suggests that, just like those other measures, ITI might be used to track processes related to emotions [18]. Previous studies with ITI demonstrate that emotion could potentially influence thermal variation in specific regions of interest. For example, feeling anxiety might lead to a decrease of temperature in the cheek region [20] while thermal variations in the nasal region, and in the maxillary region, seem to be connected to empathy [16]. However, despite its several successful applications, ITI suffers from few methodological limits. For example, the regions of interest are manually defined and there is a lack of a consistent method of analysis across studies.

\section{METHODS}

\subsection{Participants}

47 participants took part in the study $\left(\mathrm{N}_{\text {males }}=23\right)$, with a mean age of 28.5. The participants were recruited at the University of Tilburg and at the Technical University of Eindhoven. The data were collected in two sessions, the first one involved 29 participants while the second one 18 . The experiment could not involve more participants due to the outbreak of COVID-19. Participants did not wear glasses during the experiment and were asked to keep their hair away from their face in order not to create artifacts in the thermal recording. During the 15 minutes before the beginning of the experiment each participant filled in the informed consent and acclimatized to the temperature of the room. Then participants were informed they would be filmed using a thermal camera and a web camera located on the computer screen. Furthermore, they were also informed that none of these recordings would be made available to third-party.

\subsection{Experiment}

All experimental sessions took place at Deprez Building, where the MindLabs is located, in rooms that are kept under constant temperature $\left(22-24^{\circ} \mathrm{C}\right)$. For this reason, only a maximum of 4 people, included experimenters and participants, were allowed in the room at the same time.

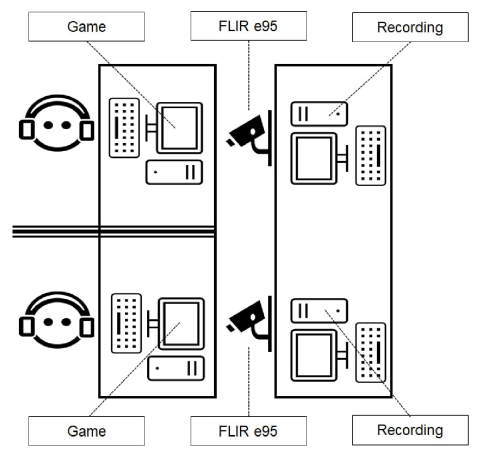

Figure 1: Overview of the experimental set-up for two participants

Two participants were seated and separated by a barrier from the rest of the room in a way that allowed them to participate in the experiment without suffering from external interference. Finally, the thermal cameras (FLIR E95) were placed about 1 meter from the participants' faces on a tripod behind the monitors. All participants wore headphones (Figure 1). The thermal recordings were started and stopped using distinct control computers placed opposite to the participants.

\subsection{Regions of Interest and Data Collection}

The experimental setup was structured to monitor the thermal variations occurring in moral decisions and non-moral decisions. Thermal variations were extracted according to 6 regions of interest defined by Berkovitz et al. [4] (Figure 2). These regions have been shown to have significant thermal variations when participants are presented with emotionally charged stimuli. Data about thermal variations in these regions was collected using two FLIR E95 thermal cameras. Actual game-play was recorded using a web camera connected to the computer used by participants. The recordings obtained with this camera were subsequently used to synchronize game-play and the thermal recordings. The thermal recordings were collected using ResearchIR, the software provided by FLIR.

\subsection{Framing Moral Decisions}

MFT was used to identify decisions in the game that were distinctly moral [15]. The first moral decision is introduced shortly after Lee, the avatar used by the participants, meets Clementine, a child who remained at home alone during a zombie apocalypse. After their encounter, Lee is forced to kill Clementine's babysitter who had become a zombie. The moral scenario is introduced when Clementine asks Lee if he killed her babysitter. In this case, the foundation triggered is the Harm/Care foundation because children are generally seen as harmless creatures needing protection and lying to Clementine is a way to avoid harming her. 


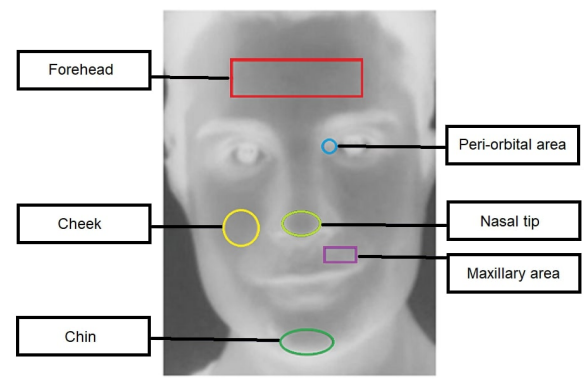

Figure 2: The regions of interest selected by Berkovitz et al. [4]

The second moral decision is presented when Lee is questioned about his connection with Clementine. The person asking the question is Shawn, a stranger who may decide to help Lee and Clementine leave the neighborhood teeming with zombies. This scenario triggers the Harm/Care foundation, due to the presence of Clementine and the possibility of keeping her safe, and the Fairness/Cheating foundation since telling Shawn the truth about the connection between Lee and Clementine might increase the likelihood of receiving help.

After having received Shawn's help, Lee and Clementine reach Shawn's farm where his father, Hershel, is waiting for him. The third moral decision is introduced when Hershel helps Lee medicate his wounded leg. Lee hurt his leg in a car accident while being escorted somewhere by a police officer. While helping Lee with his wound, Hershel asks him how he got injured. By helping Lee, Hershel may get something in return; for this reason, the Fairness/Cheating foundation is triggered, while the Loyalty/Betrayal foundation is triggered by the presence of a rival group composed of Shawn and his father Hershel. Shawn and Hershel, at this point of the video game, might consider Lee and Clementine as a rival group since they do not know their intentions and they might take advantage of Shawn and Hershel to survive the zombie apocalypse.

The fourth moral decision presented in the experiment involved Shawn and Duck, the son of a couple that stays at Shawn's house. The player has to decide who to save between the two characters while a horde of zombies approaches. This moral decision triggers the Harm/Care foundation due to the involvement of Duck, a child slightly older than Clementine, which can be saved from the potentially lethal encounter with zombies. The Loyalty/Betrayal foundation is activated since Shawn, who at this point of the game is considered a friend, is risking his life for others.

\subsection{Preprocessing}

Thermal responses occur 10 seconds after a target stimulus is presented [16]. After having extracted all the means for these, the final dataset for the analysis was created by extracting the lags between the frames. As a result, each decision presented in the game was characterized by 3 lags $(0-10$ seconds, $0-20$ seconds, and $0-30$ seconds) representing the mean variations between timestamps. This information was subsequently gathered according to the kind of decision (moral or non-moral) and the regions of interest. 20 participants were excluded from the analysis due to corrupted data or to the presence of thermal artifacts related to excessive movements or the presence of excessive facial hair [3].

\section{RESULTS}

First, an analysis of variance (ANOVA) was run to find if there was a main effect of foundations on thermal variations. Since different foundations were used to frame the moral decisions, this analysis was needed to exclude the main effect of foundations on the thermal variations. Results did show a main effect of foundations in the Nasal tip, but this effect disappeared after a Bonferroni correction $\left(\mathrm{a}_{\text {adjusted }}=.05 / 4=.0125\right)$. An Independent samples Welch's T-test was then performed for the moral and non-moral decisions at the 20 seconds lag.

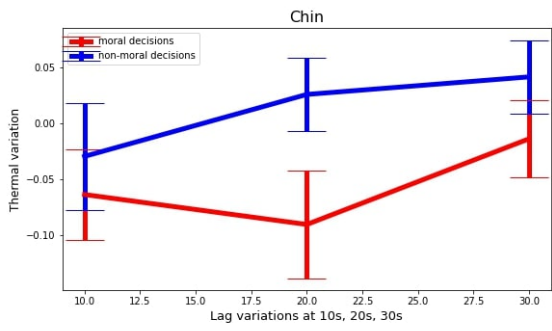

Figure 3: Mean thermal variations in chin region over time

The only significant result was in the chin region: $t(214)=2.00$, $p=.046$, with $d=.28$ (Figure 3). As shown in previous studies [16], almost all the regions of interest seem to exhibit a higher variation after 20 seconds while at 30 seconds the temperature returns to baseline. Such variation was calculated using the mean and the standard error for each ROI and then plotted as shown in Figure 3.

\section{DISCUSSION}

The effect found in this study is consistent with previous results [10], which reported a significant decrease in temperature in the chin when participants are presented with stressful stimuli. This suggests that morally charged stimuli and stressful stimuli might be similarly processed by the sympathetic system, as suggested by other research $[17,26]$. Our results are in line with a number of studies, which show that moral decisions tend to induce a higher mean variation in facial skin temperature, as compared to non-moral decisions. Most of the regions of interest examined in this study showed a decrease in temperature when subjects were presented with moral decisions. The chin region was the only region, however, in which these variations were significant, as compared with non-moral decisions, with a low effect size $(d=.28)$. Considering the general thermal variation in the entire face, this suggests that our study should be repeated with a bigger sample in order to rule out the possibility of misleading results.

If validated, these results would also give indirect evidence to the main methodological assumptions in the study. First, MFT can be used to study moral decision-making in video games. The application of MFT in this study was made possible by the presence of 
narratives and the scripted interactions with non-player characters [23]. Other theories, and especially those that are direct competitors to MFT, should be used in the future to frame moral decisions in video games to verify that this assumption is correct.

Second, ITI seems appropriately sensitive to capture both physiological responses evoked by stressful decisions and those triggered by moral decisions. In future research, ITI should be combined with other measurements to obtain a better understanding of the sympathetic activity connected to moral decision-making. More generally, ITI might be a promising method to track sympathetic activity in other domains. For example, such method might be used to track stress evoked by medical procedures without the need of any sensor in direct contact with the skin.

Nevertheless, ITI still has limitations mainly due to the lack of a shared methodology [16]. Furthermore, up to date, this is one of the first studies that explored the use of ITI in the context of video game research. Therefore, an implementation of a common methodological approach would go far to help obtain more consistent results in future studies. It should also be noted that ITI is still a developing technology and that can be affected by changes in temperature due to confounding factors [16], such as boredom, endogenous thoughts, anxiety, etc. Solving these issues might be one of the keys to future more efficacious applications of ITI to video game research.

\section{ACKNOWLEDGMENTS}

The research reported in this study is funded by the MasterMinds project, part of the RegionDeal Mid- and West-Brabant, and is co-funded by the Ministry of Economic Affairs, Region Hart van Brabant, REWIN, Region West-Brabant, Midpoint Brabant, Municipality of Breda and Municipality of Tilburg.

\section{REFERENCES}

[1] Cameron Anderson and Courtney E Brown. 2010. The functions and dysfunctions of hierarchy. Research in organizational behavior 30 (2010), 55-89. https://doi. org/10.1016/j.riob.2010.08.002

[2] Bernard W Balleine, Mauricio R Delgado, and Okihide Hikosaka. 2007. The role of the dorsal striatum in reward and decision-making. Fournal of Neuroscience 27, 31 (2007), 8161-8165. https://doi.org/10.1523/JNEUROSCI.1554-07.2007

[3] Anushree Basu, Aurobinda Routray, Suprosanna Shit, and Alok Kanti Deb. 2015 Human emotion recognition from facial thermal image based on fused statistical feature and multi-class SVM. In 2015 Annual IEEE India Conference (INDICON). IEEE, 1-5. https://doi.org/10.1109/INDICON.2015.7443712

[4] Barry Berkovitz, Claudia Kirsch, Bernard J Moxham, Ghassan Alusi, and Tony Cheesman. 2013. 3D Head and Neck Anatomy with Special Senses and Basic Neuroanatomy. [DVDROM].

[5] Karen L Blackmore, William Coppins, and Keith V Nesbitt. 2016. Using startle reflex to compare playing and watching in a horror game. In Proceedings of the Australasian Computer Science Week Multiconference. Association for Computing Machinery, 1-7. https://doi.org/10.1145/2843043.2843482

[6] Scott Clifford, Vijeth Iyengar, Roberto Cabeza, and Walter Sinnott-Armstrong. 2015. Moral foundations vignettes: A standardized stimulus database of scenarios based on moral foundations theory. Behavior research methods 47, 4 (2015), 1178-1198. https://doi.org/10.3758/s13428-014-0551-2.

[7] Vincent Conitzer, Walter Sinnott-Armstrong, Jana Schaich Borg, Yuan Deng, and Max Kramer. 2017. Moral decision making frameworks for artificial intelligence. In Proceedings of the AAAI Conference on Artificial Intelligence, Vol. 31. 4831-4835.

[8] Oliver Scott Curry, Matthew Jones Chesters, and Caspar J Van Lissa. 2019. Map ping morality with a compass: Testing the theory of 'morality-as-cooperation' with a new questionnaire. Journal of Research in Personality 78 (2019), 106-124. https://doi.org/10.1016/j.jrp.2018.10.008

[9] Burak Doğruyol, Sinan Alper, and Onurcan Yilmaz. 2019. The five-factor model of the moral foundations theory is stable across WEIRD and non-WEIRD cultures. Personality and Individual Differences 151 (2019), 109547. https://doi.org/doi.org/ 10.1016/j.paid.2019.109547

[10] Veronika Engert, Arcangelo Merla, Joshua A Grant, Daniela Cardone, Anita Tusche, and Tania Singer. 2014. Exploring the use of thermal infrared imaging in human stress research. PloS one 9, 3 (2014), e90782. https://doi.org/doi.org/10. 1371/journal.pone.0090782[

[11] John C Gibbs. 2019. Moral development and reality: Beyond the theories of Kohlberg, Hoffman, and Haidt. Oxford University Press, New York. https://doi.org/10.1093/ acprof:osobl/9780199976171.001.0001

[12] Jesse Graham, Jonathan Haidt, Sena Koleva, Matt Motyl, Ravi Iyer, Sean P Wojcik, and Peter H Ditto. 2013. Chapter Two - Moral Foundations Theory: The Pragmatic Validity of Moral Pluralism. Advances in Experimental Social Psychology 47 (2013), 55-130. https://doi.org/10.1016/B978-0-12-407236-7.00002-4

[13] Jesse Graham, Jonathan Haidt, and Brian A Nosek. 2009. Liberals and conservatives rely on different sets of moral foundations. Fournal of personality and social psychology 96, 5 (2009), 1029-1046. https://doi.org/doi.org/10.1037/a0015141

[14] Dan-Yang Gui, Tian Gan, and Chao Liu. 2016. Neural evidence for moral intuition and the temporal dynamics of interactions between emotional processes and moral cognition. Social neuroscience 11, 4 (2016), 380-394. https://doi.org/10. 1080/17470919.2015.1081401

[15] Jonathan Haidt. 2012. The righteous mind: Why good people are divided by politics and religion. Pantheon Books., New York.

[16] Stephanos Ioannou, Vittorio Gallese, and Arcangelo Merla. 2014. Thermal infrared imaging in psychophysiology: Potentialities and limits. Psychophysiology 51, 10 (2014), 951-963. https://doi.org/10.1111/psyp.12243

[17] Neil Levy, Thomas Douglas, Guy Kahane, Sylvia Terbeck, Philip J Cowen, Miles Hewstone, and Julian Savulescu. 2014. Are you morally modified?: The moral effects of widely used pharmaceuticals. Philosophy, psychiatry, and psychology 21, 2 (2014), 111-125. https://doi.org/10.1353/ppp.2014.0023

[18] Arcangelo Merla and Gian Luca Romani. 2007. Thermal signatures of emotional arousal: A functional infrared imaging study. In Annual International Conference of the IEEE Engineering in Medicine and Biology - Proceedings. IEEE, Lyon, France. https://doi.org/10.1109/IEMBS.2007.4352270

[19] Maria Serena Panasiti, Daniela Cardone, Enea F Pavone, Alessandra Mancini, Arcangelo Merla, and Salvatore M Aglioti. 2016. Thermal signatures of voluntary deception in ecological conditions. Scientific reports 6, 1 (2016), 35174. https: //doi.org/10.1038/srep35174

[20] Ioannis Pavlidis, James Levine, and Paulette Baukol. 2001. Thermal image analysis for anxiety detection. In Proceedings 2001 International Conference on Image Processing (Cat. No.01CH37205), Vol. 2. IEEE, Thessaloniki, Greece, 315-318. https: //doi.org/10.1109/ICIP.2001.958491

[21] Robert D Rogers. 2011. The roles of dopamine and serotonin in decision making: evidence from pharmacological experiments in humans. Neuropsychopharmacology 36, 1 (2011), 114-132. https://doi.org/doi.org/10.1038/npp.2010.165

[22] Joshua Rottman. 2014. Evolution, development, and the emergence of disgust. Evolutionary Psychology 12, 2 (2014), 417-433. https://doi.org/doi.org/10.1177/ 147470491401200209

[23] Malcolm Ryan, Paul Formosa, Stephanie Howarth, and Dan Staines. 2020. Measuring morality in videogames research. Ethics and Information Technology 22 (2020), 55-68. https://doi.org/10.1007/s10676-019-09515-0

[24] Malcolm Ryan, Dan Staines, and Paul Formosa. 2017. Focus, sensitivity, judgement, action: Four lenses for designing morally engaging games. Transactions of the Digital Games Research Association 2, 3 (2017), 143-173.

[25] Karen Schrier. 2017. Designing role-playing video games for ethical thinking. Educational Technology Research and Development 65, 4 (2017), 831-868. https: //doi.org/doi.org/10.1007/s11423-016-9489-7

[26] Sylvia Terbeck, Julian Savulescu, Laurence P Chesterman, and Philip J Cowen. 2016. Noradrenaline effects on social behaviour, intergroup relations, and moral decisions. Neuroscience and Biobehavioral Reviews 66 (2016), 54-60. https: //doi.org/10.1016/j.neubiorev.2016.03.031

[27] Nils-Frederic Wagner, Pedro Chaves, and Annemarie Wolff. 2017. Discovering the neural nature of moral cognition? Empirical, theoretical, and practical challenges in bioethical research with electroencephalography (EEG). Bioethical Inquiry 14 (2017), 299-313. https://doi.org/doi.org/10.1007/s11673-017-9780-2

[28] Kazufumi Yoshihara, Hiroki C Tanabe, Hiroaki Kawamichi, Takahiko Koike, Mika Yamazaki, Nobuyuki Sudo, and Norihiro Sadato. 2016. Neural correlates of fearinduced sympathetic response associated with the peripheral temperature change rate. NeuroImage 134 (2016), 522-531. 